\title{
Iron-Based Metal-Organic Frameworks as a Theranostic Carrier for Local Tuberculosis Therapy
}

\author{
Gabriela Wyszogrodzka' - Przemysław Dorożyński² (D) Barbara Gil ${ }^{3}$ - Wieslaw J. Roth ${ }^{3}$. \\ Maciej Strzempek ${ }^{3}$ - Bartosz Marszałek ${ }^{3}$ - Władysław P. Węglarz ${ }^{4}$ - Elżbieta Menaszek ${ }^{5}$. \\ Weronika Strzempek ${ }^{3} \cdot$ Piotr Kulinowski $^{6}$
}

Received: 14 February 2018 / Accepted: 3 May 2018 / Published online: 18 May 2018

(C) The Author(s) 2018

\begin{abstract}
Purpose The purpose of the study was initial evaluation of applicability of metal organic framework (MOF) Fe-MIL-101$\mathrm{NH}_{2}$ as a theranostic carrier of antituberculous drug in terms of its functionality, i.e. drug loading, drug dissolution, magnetic resonance imaging (MRI) contrast and cytotoxic safety. Methods Fe-MIL-101-NH $\mathrm{NH}_{2}$ was characterized using X-ray powder diffraction, FTIR spectrometry and scanning electron microscopy. The particle size analysis was determined using laser diffraction. Magnetic resonance relaxometry and MRI were carried out on phantoms of the MOF system suspended in polymer solution. Drug dissolution studies were conducted using Franz cells. For MOF cytotoxicity, commercially available fibroblasts L929 were cultured in Eagle's Minimum Essential Medium supplemented with 10\% fetal bovine serum.
\end{abstract}

Results MOF particles were loaded with $12 \%$ of isoniazid. The particle size $(3.37-6.45 \mu \mathrm{m})$ depended on the micronization method used. The proposed drug delivery

Przemysław Dorożyński

mfdorozy@cyf-kr.edu.pl

Faculty of Pharmacy, Department of Pharmacobiology Jagiellonian University Medical College, Medyczna 9 30-068 Kraków, Poland

2 Pharmaceutical Research Institute, Rydygiera 8 01-793 Warszawa, Poland

3 Faculty of Chemistry Jagiellonian University in Kraków, Gronostajowa 2 30-387 Kraków, Poland

4 Department of Magnetic Resonance Imaging, Institute of Nuclear Physics Polish Academy of Sciences, Radzikowskiego I 52 31-342 Kraków, Poland

5 Faculty of Pharmacy, Department of Cytobiology Jagiellonian University Medical College, Medyczna 9 30-068 Kraków, Poland

6 Faculty of Mathematics, Physics and Technical Science, Institute of Technology Pedagogical University of Cracow, Podchorążych 2 30-084 Kraków, Poland system can also serve as the MRI contrast agent. The drug dissolution showed extended release of isoniazid. MOF particles accumulated in the L929 fibroblast cytoplasmic area, suggesting MOF release the drug inside the cells. The cytotoxicity confirmed safety of MOF system.

Conclusions The application of MOF for extended release inhalable system proposes the novel strategy for delivery of standard antimycobacterial agents combined with monitoring of their distribution within the lung tissue.

KEY WORDS iron metal-organic framework (MOF) · MRI contrast agent · theranostic system · tuberculosis treatment . inhaled dosage forms

\section{ABBREVIATIONS}

$\begin{array}{ll}\text { CPMG } & \text { Carr Purcell Meiboom Gill } \\ \text { DDS } & \text { Drug delivery systems } \\ \text { DMF } & \text { Dimethylformamide } \\ \text { EDS } & \text { Element Energy Dispersive Spectroscopy } \\ \text { FOV } & \text { Field of view } \\ \text { FTIR } & \text { Fourier-transform infrared spectroscopy } \\ \text { HPMC } & \text { Hydroxypropylmethylcellulose } \\ \text { INH } & \text { Isoniazid } \\ \text { IR } & \text { Infrared } \\ \text { MCT } & \text { Mercury-Cadmium-Telluride } \\ \text { MIC } & \text { Minimum inhibitory concentration } \\ \text { MOF } & \text { Metal organic framework } \\ \text { MRI } & \text { Magnetic resonance imaging } \\ \text { MSME } & \text { Multi-Slice Multi-Echo } \\ \text { MTB } & \text { Mycobacterium tuberculosis } \\ \text { NE } & \text { Number of echoes } \\ \text { NP } & \text { Nanoparticles } \\ \text { PBS } & \text { Phosphate-buffered saline } \\ \text { PDT } & \text { Photodynamic therapy } \\ \text { SD } & \text { Standard deviation } \\ \text { SEM } & \text { Scanning Electron Microscopy } \\ & \end{array}$


TB Tuberculosis

TE Inter-echo time

TR Repetition time

WHO World Health Organization

$X R D \quad X$-ray powder diffraction

\section{INTRODUCTION}

The use of nanotechnology for medical applications is rapidly growing and is very promising in various branches of applied science (1-4). Nanoparticles (NPs) are used as diagnostic imaging agents or as drug delivery platforms, providing targeted or tissue-selective therapy, which may increase efficiency and decrease the side effects of drugs. It is also possible to combine these two functions in one particle by the design and preparation of dual-purpose nanomaterials, functioning as both diagnostic medical devices and drug delivery systems $(5,6)$. This concept of fusing diagnostics and therapy has been proposed in 2002 and called theranostics (7). Theranostic agents have been defined as "integrated nanotherapeutic systems, which can diagnose, deliver targeted therapy, and monitor the response to the therapy" (6). This integrated approach offers great opportunities in the development of personalized medicine It allows for monitoring the drug release, its biodistribution and accumulation at the target site, dose adjustment to individual patients and finally, monitoring the course of a disease $(5,8,9)$.

Freund et al. has proposed the term "atom economy" which focuses on the design of highly active materials possessing many functionalities that work together to serve a specific purpose (10). Metal-Organic Frameworks (MOFs) are excellent example to illustrate this concept and have the potential to emerge as next-generation drug delivery systems (DDS). MOFs may be of interest as carriers for theranostics, being porous structures built from inorganic nodes, which are single ions or clusters of ions, joined together by organic linkers. Such design allow to gain control over the framework architecture and, even more importantly, the pore chemistry, enabling targeted functionalization for nanomedical applications (11). Thanks to their porous structure, MOFs seem to be promising drug vehicles with potential high drug loading (12-14). The control of guest release profiles can be gained by the choice of the type of functional group of the linker and tuning of the pore size (15).

In the case of MOF application for drug delivery, they should exhibit stability under physiological condition, minimal toxicity, biodegradability and as biocompatibility for both metal and bridging linker ligand. MOFs as components of drug delivery system are discussed in the context of other nanoparticulate carriers (mesoporous silica, dendrimers) in the work by Wuttke et al. (16). Safety of MOF as drug delivery nanomaterials varies strongly with effector cell type.
Therefore, it is necessary to evaluate their nanosafety regarding particular application and involved cell type. Wuttke et al. (11) discuss the effects of MIL-101(Cr) and MIL-101(Fe) on human endothelial and mouse lung cells, a first line of defense upon systemic blood-mediated and local lung-specific applications of nanoparticles.

Magnetic resonance imaging (MRI) has become a powerful tool in medicine for non-invasive imaging of the internal structure and functions of living organisms as well as local properties of tissues (17). Magnetic nanoparticles may be applied as contrasting agents providing either negative $\left(\mathrm{T}_{2}\right.$-weighted $)$ or positive imaging contrast $\left(\mathrm{T}_{1}\right.$-weighted) (18). A potential MRI contrasting agent has to fulfill several requirements related to tolerance, safety, toxicity, stability, osmolarity, viscosity, biodistribution, elimination, and metabolism (19).

Embedding paramagnetic cations $\left(\mathrm{Gd}^{3+}, \mathrm{Mn}^{2+}, \mathrm{Fe}^{3+}\right)$ in MOF structure make them possible to be used as MRI contrast agents. Among them, iron is the best option from a toxicological point of view. Nanoscale iron MOFs (MIL-53, MIL-88A, MIL-88Bt, MIL-89, MIL-100 and MIL101_ $\mathrm{NH}_{2}$ ), with engineered cores and surfaces, have been shown to be able to serve as drug carrier and magnetic resonance contrast agent according to good ability for modification of relaxivities (20).

Polymeric surface allows for the MOF structures modification to implement properties such as increased chemical and colloidal stability which enhance the cellular uptake, or dyelabeling, which enables for example, the investigation of nanoparticle uptake into tumor cells by fluorescence microscopy (21). It has been proved, that coated iron MOFs can retain their MRI contrast properties. MOF NPs are frequently coated in order to prevent leakage of the drug before they reach the target (e.g. exosome-coated MIL-88A, liposome coated MIL-88A) $(21,22)$. MOFs, including iron MOFs, can be (multi)functionalized (example can be found in the work of Roeder et al. 2017 (21)) - molecular units can be anchored on the outer surface of MOFs.

MOFs can be used as stimuliresponsive DDS for cancer therapy (targeted chemotherapy, gene therapy). Recent studies also shown that it is possible to develop MOF based delivery systems for photodynamic therapy (PDT) of cancer (23).

While theranostics has been intended mainly for cancer treatments, there are numerous other therapeutic targets for which the effectiveness of therapy could be increased by local drug delivery and monitoring of its distribution. Among them tuberculosis (TB) seems to be especially important.

$\mathrm{TB}$ is one of the top three infectious diseases - together with HIV and malaria - causing morbidity and death worldwide. According to the World Health Organization (WHO) estimations, about $30 \%$ of the world's population is infected with Mycobacterium tuberculosis (MTB). Every year, about 10 million of new cases are registered and about 1.5 to 2 million of deaths are caused by TB, according to the report by the 
WHO agency (24). TB infections frequently become multidrug-resistant, since the conventional treatment protocol is based on an antibiotic therapy carried out over periods of 6 to 9 months (25).

Isoniazid (Isonicotinic Acid Hydrazide, INH) is particularly suitable for use as a model drug in theranostic drug delivery because it is an antibiotic used as a first-line agent in the prevention and treatment of both latent and active tuberculosis. It is effective against mycobacteria, especially Mycobacterium tuberculosis since it inhibits biosynthesis of the mycolic acid. Oral administration of INH and long-term therapy causes several serious side effects, which could even force treatment discontinuation. INH is known to cause hepatitis (26), hepatic injury and neuropsychiatric disturbances (27) including, among others, uncontrollable seizures (28) or polyneuropathy (29). The conventional oral route of INH administration causes periodic decrease of its concentration below the effective minimum inhibitory concentration (MIC), allowing MTB bacilli to develop resistance (30). The distribution of the antituberculous drug within the infected tissue is equally important. The studies of Kjellsson et al. (31) on a TB infected animal model have shown that after systemic administration the distribution of isoniazid, rifampicin and pyrazinamide in the lung tissue is uneven. The concentrations of drugs in pulmonary lesions where the pathogen is located have been markedly lower than in the surrounding lung tissue.

Hickey et al. (32) indicated the problem of dramatic increase in extremely drug-resistant TB around the world and highlighted that pulmonary administration offers the ability to deliver drug directly to the infected macrophages in the deepest part of lungs. Comparison of various drypowders containing anti-TB drugs for inhalation presented by Pham et al. (33) indicates that the strategy of inhaled therapy is the main and the most promising alternative to traditional approach and is necessary to achieve global TB control.

The MOF-based theranostics may be an interesting alternative for the standard therapy of tuberculosis. Pulmonary route should ensure high local drug concentration (avoiding side effects of the systemic drug action). Moreover, possibility to generate contrast in magnetic resonance images should allow deposition and lung clearance monitoring (34-36) the first tests on animals were performed in a clinical system with a clinical nebulization setup and a low inhaled dose (34). There are two reasons for deposition monitoring: optimization of the dosage form at formulation stage and optimization of therapy.

The aim of the study was initial evaluation of applicability of MOF Fe-MIL-101- $\mathrm{NH}_{2}$ as a theranostic carrier of antituberculous drug in terms of its functionality, i.e. drug loading, drug dissolution, MRI contrast and cytotoxic safety.

\section{MATERIALS AND METHODS}

\section{MOF Synthesis and Drug Incorporation}

Fe-MIL-101- $\mathrm{NH}_{2}$ was synthesized according to the procedure reported by Bauer et al. (37). The MOF powder samples were comminuted by milling in an agate ball mill for $24 \mathrm{~h}$ and then sonicated for $5 \mathrm{~min}$. Using a CP $130 \mathrm{~PB}(130 \mathrm{~W}, 20 \mathrm{kHz})$ ultrasonic processor (Cole-Parmer, USA), at 70\% of maximum amplitude. After drying MOF was activated under vacuum at $100^{\circ} \mathrm{C}$ for $30 \mathrm{~min}$.

Isoniazid (Sigma-Aldrich, Germany) was incorporated into the MOF matrix by mixing $1.5 \mathrm{~mL}$ of saturated solution of INH in DMF with $300 \mathrm{mg}$ of micronized Fe-MIL-101-- $\mathrm{NH}_{2}$. The slurry was mixed for $12 \mathrm{~h}$ at room temperature and the product was separated by centrifugation and washed with ethanol.

\section{MOF Characterisation}

The characterization by X-ray powder diffraction was carried out using a Bruker AXS D8 Advance (Bruker, Germany) diffractometer in the range $1-30^{\circ} 2 \Theta$ using $\mathrm{CuK} \alpha(\lambda=$ $0.154178 \mathrm{~nm})$ radiation.

Infrared (IR) spectra were measured in transmission mode using Tensor 27 FTIR spectrometer (Bruker, Germany) equipped with an MCT (Mercury-Cadmium-Telluride) detector at spectral resolution of $2 \mathrm{~cm}^{-1}$. Before measurement a sample was deposited on an IR-transparent silicon wafer (pure for electronic purposes) by placing its ethanol solution directly on the disc and evaporating the solvent. The wafer was placed in an IR cell with $\mathrm{KBr}$ windows and slowly heated under constant pumping $\left(10^{-3} \mathrm{Torr}\right)$ to $50^{\circ} \mathrm{C}$ (with the drug present) or $100^{\circ} \mathrm{C}$ (pure MOF).

Scanning Electron Microscopy (SEM) analysis was performed using Nova Nano SEM 200 (FEI Europe B.V.) cooperating with the Element Energy Dispersive Spectroscopy (EDS) analyzer (EDAX Inc., U.S.A.) using secondary electrons in low vacuum conditions $(60 \mathrm{~Pa})$. Samples of MOF without treatment, MOF after milling and MOF after milling and sonication were analyzed.

The particle size distribution of the powder samples was measured in terms of particle diameter at $50 \%$ in the cumulative distribution (Dx (38)) using laser diffraction particle size analyzer Mastersizer 3000 (Malvern Instruments Ltd., United Kingdom).

\section{Drug Release}

The drug release study was performed in Franz cells (39). The donor compartment was separated from the acceptor compartment by a cellulose acetate filter with pore size of $0.8 \mu \mathrm{m}$ (Sartorius, Germany) covered with $200 \mu \mathrm{L}$ of $1 \%$ 
mucin solution simulating the mucus layer deposited on lung epithelium (40-43). The $5 \mathrm{mg}$ of composite powder was placed in the donor compartment. The acceptor compartment was filled with $5 \mathrm{~mL}$ of phosphate-buffered saline (PBS) at pH $7.4(44,45)$. The experiment was performed at $37^{\circ} \mathrm{C}$ under continuous stirring at $140 \mathrm{rpm}$. Samples of $0.4 \mathrm{~mL}$ were taken from the acceptor compartment at 0.5 , $1,1.5,2,3,4,5,6,8,10,24$ and $48 \mathrm{~h}$ and were replaced with $0.4 \mathrm{~mL}$ of a fresh medium. A blank was carried out by evaluation of released crystalline isoniazid in quantity corresponding to the content of drug in MOF. The isoniazid concentration was analyzed in an aqueous solution using a UV-Vis spectrophotometer UV 1800 (Shimadzu, Japan) at a wavelength $\lambda=262.0 \mathrm{~nm}$.

\section{Magnetic Resonance Imaging and Relaxometry}

Nuclear magnetic resonance imaging and relaxometry were performed using 9.4 T MRI research system (Bruker Biospin, Germany) and TopSpin 2.0 software (Bruker Biospin, Germany). For this purpose 0.3, 1.7, 3.3, 6.7, 13.3, 26.7 and $53.3 \mathrm{mg} / \mathrm{mL}$ MOF suspensions in $2 \%$ hydroxypropylmethylcellulose (HPMC; Metolose, 90SH, $400 \mathrm{cP}$ - Shin-Etsu, Japan) water solution were prepared.

$\mathrm{T}_{1}$ and $\mathrm{T}_{2}$ relaxation times were measured using Inversion Recovery and Carr Purcell Meiboom Gill (CPMG) pulse sequences, respectively - number of accumulations $(\mathrm{NA})=8$ and dwell time $(\mathrm{DW})=10 \mu \mathrm{s}$. For $\mathrm{T}_{1}$ assessment separate measurements with 16 logarithmically spaced inversion time values were performed in order to sample $T_{1}$ recovery. For CPMG sequence the signal was acquired after 16 logarithmically spaced $n \bullet$ TE time intervals (where TE $=0.2 \mathrm{~ms}$ ). The data were fitted assuming monoexponential $\mathrm{T}_{2}$ decay $\left(\mathrm{T}_{1}\right.$ recovery). Linear regression of $\mathrm{R}_{1}\left(=1 / \mathrm{T}_{1}\right)$ and $\mathrm{R}_{2}\left(=1 / \mathrm{T}_{2}\right)$ vs. MOF concentration in suspension was performed to obtain $r_{1}$ and $r_{2}$ relaxivities with intercept set at $R_{1}$ and $R_{2}$ values of pure polymer solution.

MR imaging was performed using Multi-Slice Multi-Echo (MSME) imaging sequence for pure 2\% HPMC solution as well as for 26.7 and $53.3 \mathrm{mg} / \mathrm{mL}$ MOF suspensions in $2 \%$ HPMC solution. Two sets of images at two different repetition time (TR), i.e. $0.7 \mathrm{~s}$ and $3 \mathrm{~s}$ were obtained with following parameters: field of view $(\mathrm{FOV})=28 \times 28 \mathrm{~mm}^{2}$, slice thickness $=1 \mathrm{~mm}$ (axial cross section), image matrix size of $256 \times$ 256 , number of echoes $(\mathrm{NE})=256$, inter-echo time $(\mathrm{TE})=$ $3.5 \mathrm{~ms}, \mathrm{NA}=2$.

\section{In Vitro Cytotoxicity}

For MOF cytotoxicity study, commercially available fibroblasts L929 (Sigma-Aldrich, Germany) were cultured in Eagle's Minimum Essential Medium supplemented with $10 \%$ fetal bovine serum (ATCG, USA). Cells were maintained at $37^{\circ} \mathrm{C}$ in a humidified incubator (ThermoSci, Germany) with 5\% $\mathrm{CO}_{2}$ until 70-80\% confluence was obtained. At passage 3 the cells were detached using 0.5\% trypsin-EDTA, centrifuged and suspended in the fresh growth medium. Next, the cells were seeded at density $0.5 \cdot 10^{4}$ cells/200 $\mu \mathrm{L}$ in 96-well culture plates (Nunc, Denmark) and allowed to adhere. After $24 \mathrm{~h}$, suspensions of milled or milled and sonicated particles of Fe-MIL-101- $\mathrm{NH}_{2}$ were added to the culture at two concentrations: 0.625 and $1.25 \mathrm{mg} / \mathrm{mL}$.

Viability of L929 fibroblasts cultured in contact with MOF suspension for 24 or $72 \mathrm{~h}$ was determined with resazurinbased reagent PrestoBlue ${ }^{\mathrm{TM}}$ (Invitrogen, USA). Fluorescent product of the reaction was detected using POLARstar Omega microplate reader (BMG Labtech, Germany). Obtained results are presented as the mean \pm standard deviation (SD) of five samples. Significant effects $(p<0.05)$ were determined using Student's $t$-test. Cells morphology was observed under contrast phase inverted microscope CKX53 (Olympus, Japan).

\section{RESULTS AND DISCUSSION}

\section{Synthesis and Characterization of MOFs.}

Fe-MIL-101- $\mathrm{NH}_{2}$ of good quality was synthesied, as confirmed by the diffractogram (XRD pattern) (Fig. 1), which showed a very good agreement with the calculated MIL-101 patterns published in the literature (46). The intensities of the reflections increased considerably after washing with ethanol of both the as-synthesized sample, containing free DMF molecules, and for the MOF sample with incorporated INH. This is most likely due to the common effect observed when micropores are emptied from the occluded guest molecules.

Iron-containing MOF compound Fe-MIL-101- $\mathrm{NH}_{2}$ was chosen for two reasons. First, it is one of the most stable Febased Metal-Organic Frameworks which has been already reported as a carrier for bioactive $(47,48)$ or magnetic $(49,50)$ compounds. Second, the presence of the $-\mathrm{NH}_{2}$ functional groups in the linker allows easy determination whether guest molecules are located inside the MOF cavities, interacting with them and changing their properties, or are only adsorbed in the intercrystalline voids.

MIL-101 has a rigid zeotype (MTN) crystal structure (50) with two types of cages. Its medium size cavities with the diameter of $2.9 \mathrm{~nm}$ are accessible through pentagonal windows with the opening of $1.2 \mathrm{~nm}$, while large $3.4 \mathrm{~nm}$ cavities have hexagonal windows with the diameter of $1.6 \mathrm{~nm}(51)$. The 3D molecular size for INH was determined (52) using Chem Office Software 2008 as equal to $1.05 \times 0.72 \times$ $0.31 \mathrm{~nm}$. The spacious MOF cavities together with relatively large apertures make carrier suitable for incorporation of bulky drug molecules. 


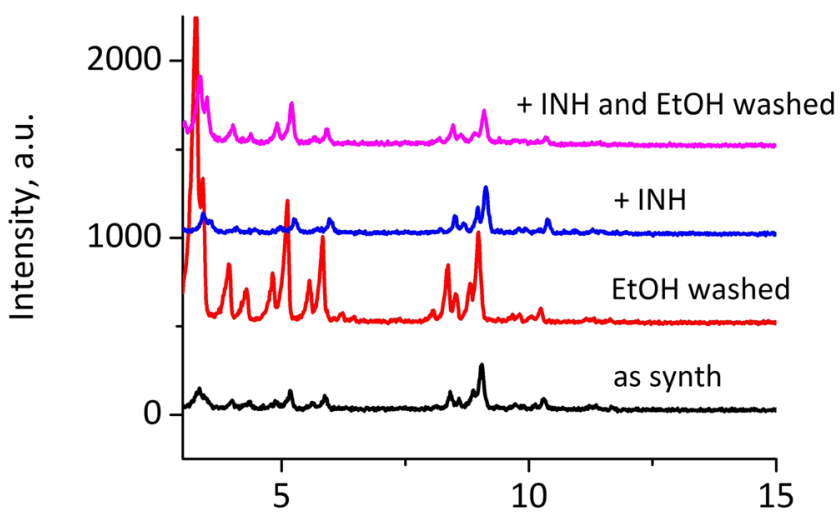

$2 \Theta$, deg

Fig. I XRD patterns of Fe-MIL-I I I- $\mathbf{N H}_{\mathbf{2}}$ : as synthesized, washed with ethanol, after introduction of isoniazid and again washed with ethanol after isoniazid introduction (from bottom to top)

\section{Particle Size}

Regarding particle size, according Hirschle et al. (53), MOF NPs intended to use as drug delivery systems shoud be characterized using multiple techniques - as powder and also in dispersion. They also discuss the appropriate method for obtaining the nanoparticle size that is meaningful in the context of the desired application. In our work we evaluated the impact of milling and subsequent ultrasonication on Fe-MIL$101-\mathrm{NH}_{2}$ crystal size and shape. Three kinds of samples were examined: MOF without treatment (Fig. 2a), MOF after milling (Fig. 2b) and MOF after milling and ultrasonication (Fig. 2c).

For dry powders SEM imaging and measurement of particle size distribution were carried out.

Milling the samples did not change the overall shape and size of crystals, which appeared to be undamaged in SEM images (Fig. 2b). It was also not able to break crystal aggregates; thus this method cannot be used by itself for micronization of the samples. For this reason, crystals after milling were ultrasonicated, which caused breakage of the aggregates resulting in very homogeneously looking SEM images of single crystals, as shown in Fig. 2c.

The measurement of particle size distribution was performed using laser diffraction method. The $\mathrm{Dx}(38)$ parameter for MOF samples without treatment was $6.45 \mu \mathrm{m}$ (SD: \pm 0.20). Dx (38) decreased as a result of milling to $5.51 \mu \mathrm{m}$ (SD: \pm 0.21$)$ and to $3.37 \mu \mathrm{m}$ (SD: \pm 0.03 ) after subsequent sonication. It indicates the possibility to adjust Fe-MIL-101$\mathrm{NH}_{2}$ size by applying the appropriate micronization method to obtain the desired size.

\section{INH Incorporation}

To evaluate the content of the drug inside the pores the XRD study was conducted, and no free isoniazid crystals were evident in the XRD pattern (Fig. 1). The intensities of all XRD reflections decreased markedly for the isoniazid-containing MOF, almost to the same level as observed for the assynthesized samples filled with the solvent (DMF). Washing the drug-MOF composite with ethanol caused partial extraction of the encapsulated isoniazid, which was indicated by increased intensities of the XRD reflections. This suggests that INH molecules were located inside the pores of the MOF material.

The mode of INH incorporation was further investigated by FTIR spectroscopy (Fig. 3). After INH introduction the spectrum was not a simple superposition of the spectra chearchterstic of the pure component of MOF and INH, again suggesting that the drug molecules were located inside the pores of Fe-MIL-101-NH $\mathrm{NH}_{2}$. In the pure MOF material the $-\mathrm{NH}_{2}$ groups of the structure-forming linker were characterized by two IR maxima at 3505 and $3390 \mathrm{~cm}^{-1}$ characteristic of $\mathrm{v}_{\mathrm{as}}$ and $\mathrm{v}_{\mathrm{sym}} \mathrm{N}-\mathrm{H}$ vibrations $(38,54)$, each split into two components - one at the higher and one at the lower frequency. The lower frequency components (bands at 3485 and $3295 \mathrm{~cm}^{-1}$ ) could be due to hydrogen-bonding of the $-\mathrm{NH}_{2}$ moieties. This would suggest that $-\mathrm{NH}_{2}$ functionalities of the
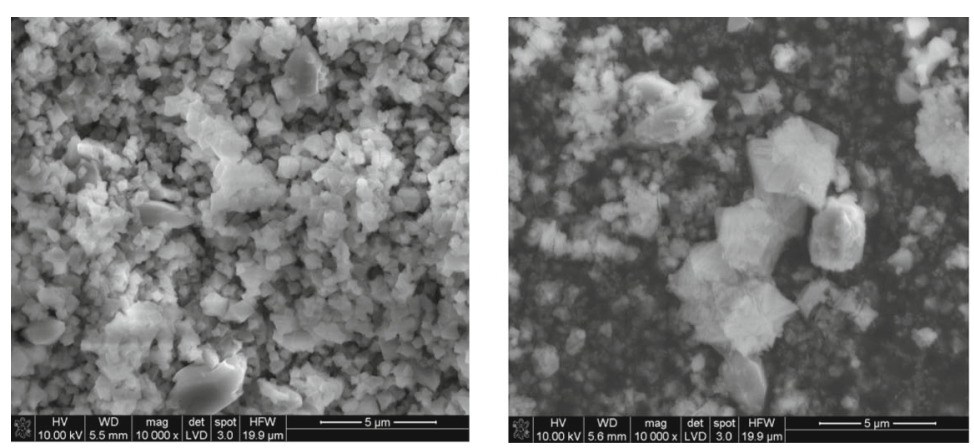

$$
\begin{gathered}
\mathrm{Dx}(50)=6.45 \mu \mathrm{m}(\mathrm{SD}: \pm 0.20) \\
\text { a. Fe-MIL-101- } \mathrm{NH}_{2}
\end{gathered}
$$

$\mathrm{Dx}(50)=5.51 \mu \mathrm{m}(\mathrm{SD}: \pm 0.21)$

b. Fe-MIL-101- $\mathrm{NH}_{2}-\mathrm{M}$

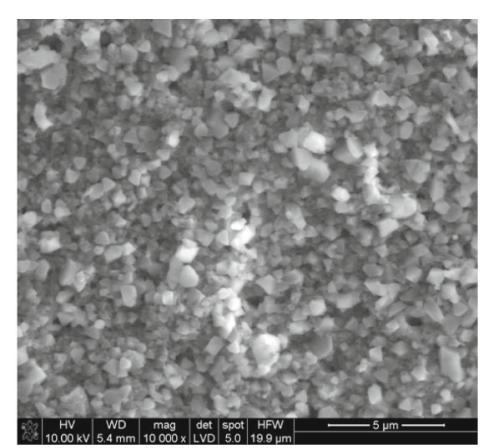

$\operatorname{Dx}(50)=3.37 \mu \mathrm{m}(\mathrm{SD}: \pm 0.03)$

c. Fe-MIL-101- $\mathrm{NH}_{2}-\mathrm{MU}$

Fig. 2 SEM images of Fe-MIL-IOI-NH $\mathbf{N}_{\mathbf{2}}$ samples: (a) without treatment, (b) after milling (-M), (c) after milling and ultrasound treatment (-MU) 
Fig. 3 Drug loading: IR spectra in the $\mathrm{N}-\mathrm{H}$ stretching vibration region (left) and framework vibrations (right) in transmission mode of: pure isoniazid (a), pure Fe-MILI $0 \mathrm{I}-\mathrm{NH}_{2}(\mathbf{b})$, physical mixture of Fe-MIL-IOI-NH $\mathrm{NH}_{2}$ with isoniazid (c), and isoniazid incorporated into FeMIL-I $0 \mathrm{I}-\mathrm{NH}_{2}$ (d). Arrows show the red-shift of some of the IR maxima

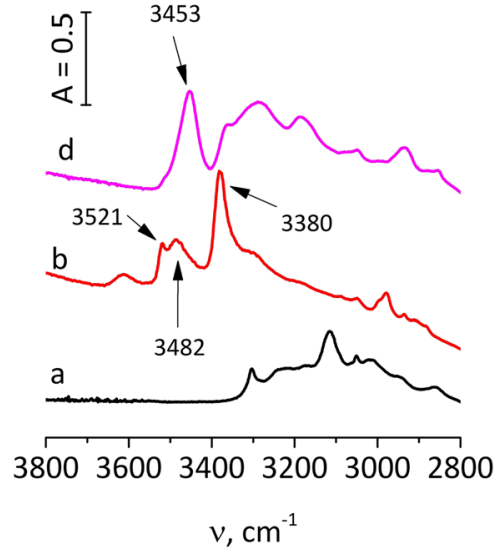

linker may be present both as free and the intramolecular hydrogen-bonded species even in the absence of DMF molecules. After INH introduction, the IR maxima characteristic of free $-\mathrm{NH}_{2}$ disappeared due to formation of new hydrogen bonds with the isoniazid molecules. The INH molecules were concluded to interact via its carbonyl groups because this particular maximum was much wider in the spectrum of the composite than in the pure, crystalline isoniazid (Fig. 3b, band at $1664 \mathrm{~cm}^{-1}$ ) and of lower frequency than expected from vibrations of the free $\mathrm{C}=\mathrm{O}$ bond. The spectral characteristics of isoniazid also changed considerably. The most important changes were these in the ring breathing vibrations (Fig. 3b, band at $995 \mathrm{~cm}^{-1}$ ) - this band red-shifted (by $30 \mathrm{~cm}^{-1}$ ) and its intensity decreased. Such changes may be assigned to socalled confinement effect, resulting in constrained breathing of the aromatic ring inside the MOF pores. Similar changes, also in the $1800-1300 \mathrm{~cm}^{-1}$ region, were observed upon incorporation of INH into the montmorillonite and saponite clays (55). From the IR results it can be deduced that INH molecules were located inside the pores of Fe-MIL-101- $\mathrm{NH}_{2}$, strongly interacting by hydrogen bonding via carbonyl groups with the $-\mathrm{NH}_{2}$ functionalities of the organic linker. Such strong interaction may result in the slower release of the INH.

The study of total INH content by mixing in water during $12 \mathrm{~h}$ revealed that INH constituted $12 \%$ (SD: \pm 0.8 ) of the composite mass. This amount was considered as $100 \%$ of the isoniazid content in the release study.

\section{INH Dissolution}

After first 6 h of dissolution inside the Franz cell 55.0\% (SD: \pm 5.2 ) of the isoniazid content was released from the composite powder, it reached $89.3 \%$ (SD: \pm 1.2 ) after $24 \mathrm{~h}$ and $94.2 \%$ (SD: \pm 4.3 ) after $48 \mathrm{~h}$. No burst effect was observed. Dissolution of crystalline isoniazid, not incorporated in MOF structure, was much faster and after $3 \mathrm{~h}$ (94.6\%; SD: \pm 6.6) no significant increase in the amount of released drug was observed (Fig. 4). These results allowed the conclusion that MOF can act as isoniazid carrier for extended release.

Moreover, it has been also shown, that varying $\mathrm{NH}_{2}$ to $\mathrm{C}_{4} \mathrm{H}_{4}$ linker ratio for MIL-101(Fe) it is possible to obtain continuum of guest molecules binding energy states (representing specific interaction with guest molecules) and in consequence to tune release profile. (15)

INH dissolution requires more extensive discussion. To date there are no pharmacopoeial dissolution method for inhalatory formulations and no single in vitro method has emerged as the ideal choice for performing dissolution tests and to estimate in vivo solubility in the lung fluids $(56,57)$. The reason is that lungs have unique features that are difficult to replicate in vitro, such as extremely small amount of aqueous fluid and the presence of lung mucus and surfactant (56-59).

In the current study, similar setup was used as it has been previously presented, for example, by Kim et al. (39). Due to the physiology of the lung and the relatively low water content in the respiratory tract, the $5 \mathrm{~mL}$ Franz cells allow in vitro approach in comparing the drug release profiles of inhalation dry powder formulations $(60,61)$. Highly viscous mucus is a major obstacle for particles to reach the respiratory airway

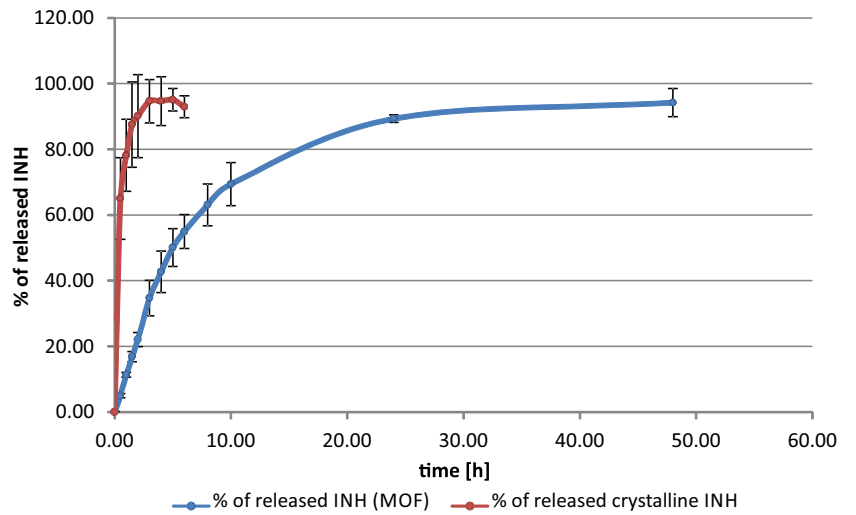

Fig. 4 Isoniazid dissolution: Comparative in vitro dissolution (drug release) profile of INH from Fe-MIL- I0 I-NH 
(41) thus, similarly to Terzano et al. (40) in our study the mucin solution imitating mucus barrier was used.

Observation made by May et al. (62) revealed that dissolution profiles obtained in Franz cell never reached more than $90 \%$ of recovery rate which might be caused by not homogenous contact area to dissolution medium under membrane due to small air bubbles or wrinkles in the membrane. Similarly, in our study incomplete dissolution ( 95\%) can also be observed for both crystalline isoniazid powder and INH incorporated in MOF.

\section{Nuclear Magnetic Resonance Relaxometry and Magnetic Resonance Imaging}

The spin-lattice relaxation rates $\left(1 / \mathrm{T}_{1}\right.$ or $\left.\mathrm{R}_{1}\right)$ and spin-spin relaxation rates $\left(1 / \mathrm{T}_{2}\right.$ or $\left.\mathrm{R}_{2}\right)$ versus concentration of $\mathrm{MOF}$ in the suspensions are presented in Fig. 5a. The relaxation rates $R_{1}$ and $R_{2}$ increased linearly with the concentrations of suspended material $\left(R^{2}=0.9926\right.$ and $R^{2}=0.9948$, respectively). The relationships between relaxation rates and concentration of Fe-MIL-101- $\mathrm{NH}_{2}$ in the suspensions were found to be equal to:

$\mathrm{R}_{\mid, 2}=\mathrm{r}_{\mid, 2} \mathrm{G}+\mathrm{i}_{\mid, 2}$

Where $\mathrm{C}$ is the concentration of Fe-MIL-101- $\mathrm{NH}_{2}$ in the suspension, expressed in $\mathrm{mg} / \mathrm{mL}$. The values of relaxivity $r_{1}=2.4(\mathrm{mg} / \mathrm{mL})^{-1} \mathrm{~s}^{-1}$ and intercept $i_{1}=0.4 \mathrm{~s}^{-1}$ for $\mathrm{T}_{1}$ relaxation while $r_{2}=22.6(\mathrm{mg} / \mathrm{ml})^{-1} \mathrm{~s}^{-1}$ and intercept $i_{2}=$ $0.64 \mathrm{~s}^{-1}$ for $\mathrm{T}_{2}$ relaxation were found, respectively. These results suggested that Fe-MIL-101- $\mathrm{NH}_{2}$ could be used as an effective contrast agent.

To demonstrate the possibility to use Fe-MIL-101- $\mathrm{NH}_{2}$ as an effective contrast agent, MR images were obtained using multi-echo pulse sequence with inter-echo time of $3.5 \mathrm{~ms}$ and with two different repetition times, i.e. 0.7 and $3 \mathrm{~s}$. Therefore, two sets of images were obtained. Images demonstrating positive and negative contrasts were chosen from these two image sets, and are presented in Fig. 5b. Sample No. 1 was a 2\% HPMC solution. Two other samples were 26.7 and $53.3 \mathrm{mg} / \mathrm{ml}$ MOF suspensions in $2 \%$ HPMC solution, i.e. samples No. 2 and 3 respectively.

When working with short repetition time of $0.7 \mathrm{~s}$ at the $1 \mathrm{st}$ echo (3.5 ms), positive contrast was obtained (Fig. 5b, left image). In this case, the image intensity for samples No. 2 and No. 3 was higher than for sample No. 1 and it increased with $\mathrm{MOF}$ concentration. An image obtained at the 2 nd echo (echo time of $7 \mathrm{~ms}$ ) of this image set also demonstrated positive contrast compared to the reference sample, however the difference in image intensity between samples No. 2 and No. 3 was negligible (data not presented). When working with the long repetition time of $3 \mathrm{~s}$ (Fig. 5b, right image) negative contrast was achieved. As an example, the image obtained at 6th echo (echo time of $21 \mathrm{~ms}$ ) is presented. In this case, image intensity for samples No. 2 and No. 3 was lower than for reference (sample No. 1 - pure polymer solution) and it decreased with increasing MOF concentration.

Only small number of ex vivo and one in vivo study showed that regional distribution/deposition of aerosol, containing MRI contrast agents (iron oxide, Gd-DOTA) in rat lungs can be successfully monitored using MRI $(34,35)$. The results of these studies suggest that the approach to combine drug delivery with contrast agent (theranostic) is promising for such demanding application.

\section{In Vitro Viability/Cytotoxicity}

The viability of fibroblasts cultured for $24 \mathrm{~h}$ with both concentration of Fe-MIL-101-NH $\mathrm{NH}_{2}(0.625$ and $1.25 \mathrm{mg} / \mathrm{mL}) \mathrm{did}$ not differ between samples and between samples and the control (Fig. 6). No cytotoxic effect of MOF was observed for this series. After $72 \mathrm{~h}$ the viability of cells cultured with $\mathrm{MOF}$ samples was significantly $(p<0.05)$ lower in comparison to the control, but the number of cells was still higher than observed after $24 \mathrm{~h}$, which means that the addition of MOF did not inhibit their proliferation. It was also shown that micronization of MOF crystals did not influence their cytotoxicity.

The morphology of L929 fibroblasts after $24 \mathrm{~h}$ culture with the addition of two concentrations of milled or milled and sonicated particles of Fe-MIL-101- $\mathrm{NH}_{2}$ is presented in Fig. 6b. In the case of $1.25 \mathrm{mg} / \mathrm{mL}$ MOF concentration, the cells were eclipsed with the particles. MOF particles in lower concentration $(0.625 \mathrm{mg} / \mathrm{mL})$ were mostly phagocytosed and were clearly visible inside the cells. It proved that Fe-MIL-101-NH particles could release drug inside cells. Tubercle bacilli after reaching the alveoli are phagocytosed and accumulate in alveolar macrophages to form tubercles. It implies that delivery of isoniazid directly to the cell increase the effectiveness of therapy. In our study, no damage in L929 cells' morphology (shape and appearance) after treatment was observed. Majority of cells had elongated shape, characteristic for fibroblast that proved the cells' viability. Cytotoxicity results described above are the first results for Fe-MIL-101- $\mathrm{NH}_{2}$ - to the best of our knowledge such study has not been published previously. Moreover, studies on MOF materials toxicity are scarce. The benefits of MOF miniaturization, apart from their proven effectiveness in cellurar uptake, defined their in viwo fate and consequently, their toxicity/activity (63).

Wuttke et al. conducted cytotoxicity study for MIL-100(Fe) and MIL-101(Cr) nanoparticles with and without lipid (1,2dioleoyl-sn-glycero-3-phosphocholine) layer on human endothelial cells (HUVEC and HMEG), alveolar epithelial cells (MLE12) and mouse alveolar macrophages (MH-S). Results revealed that both MOFs are well tolerated by endothelial cells whereas the MIL-100(Fe) with a lipid layer caused some 
Fig. 5 Nuclear Magnetic Resonance Relaxometry and

Magnetic Resonance Imaging:

(a) The quantitative linear correlation between relaxation rates $\left(R_{1}, R_{2}\right)$ and concentration of FeMIL-IOI- $\mathrm{NH}_{2}$ in a suspension. (b) MR images of FeMIL-IOI- $\mathrm{NH}_{2}$ showing the examples of positive (left image) and negative (right image) contrast due to differences in imaging sequence parameters $(\mathrm{TR}=0.7 \mathrm{~s}, \mathrm{TE}$ $=3.5 \mathrm{~ms}$ and $\mathrm{TR}=3 \mathrm{~s}$, TE

$=21 \mathrm{~ms}$ respectively)
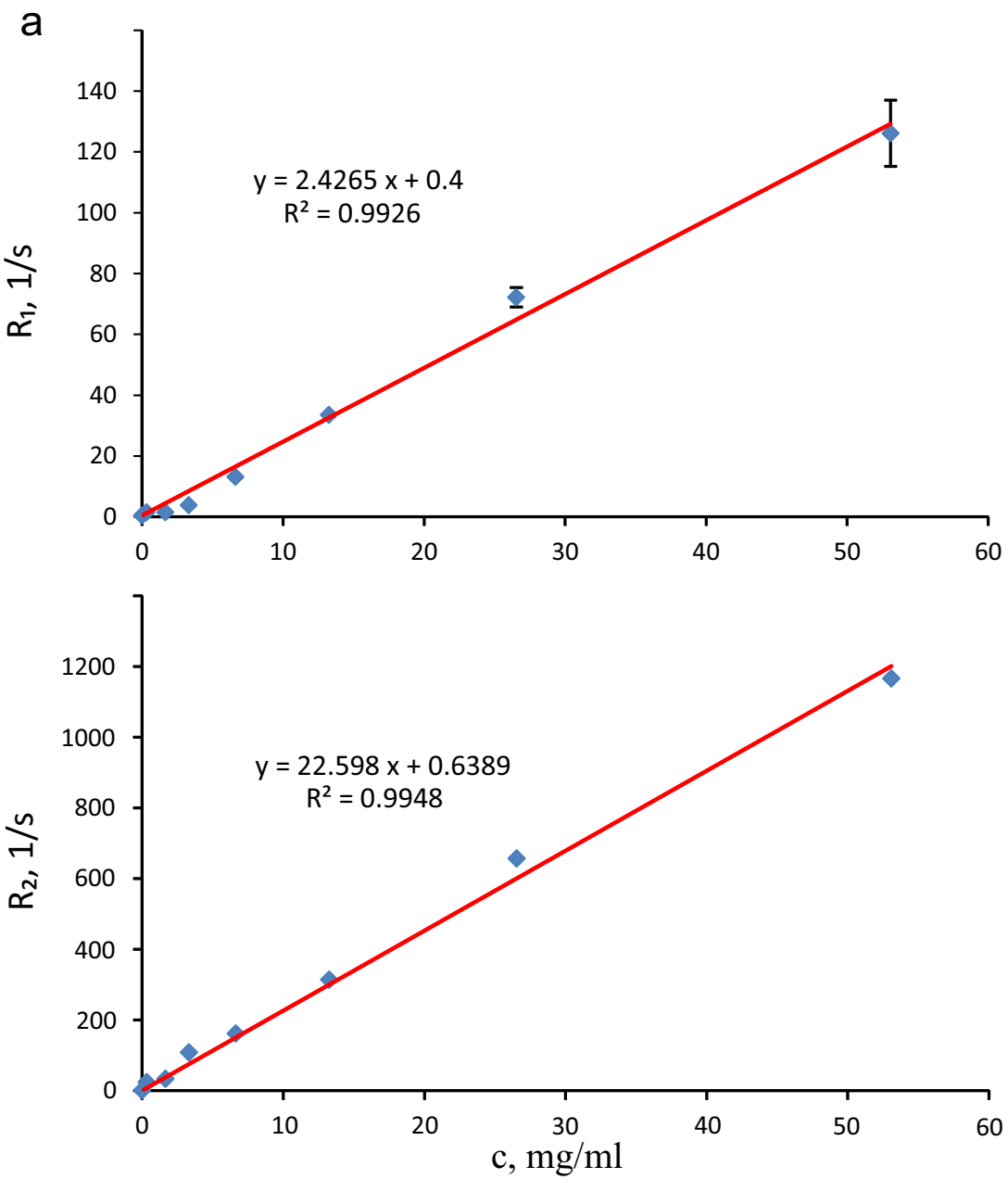

b

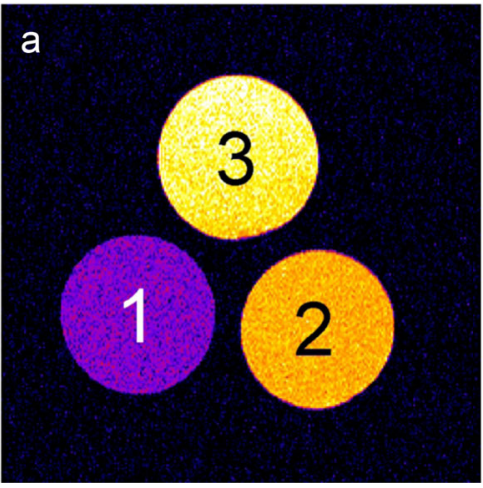

apoptotic cell death. Alveolar epithelial cells tolerate only lipid-coated MOF at lower doses of up to 50-100 $\mu \mathrm{g} \mathrm{mL}-1$. Alveolar macrophages appear to be particularly sensitive to iron MOF, which cause pronounced induction of a cellular stress response.

Grall et al. (64) have recently investigated in vitro cell toxicity of Fe-MIL-100 nanoparticles and their $\mathrm{Cr}$ and $\mathrm{Al}$ analogue nanoparticles on lung (A549 and Calu-3) and hepatic (HepG2 and Hep3B) cell lines. Authors proved that pulmonary, ingestion or intravenous exposure modes were not toxic to the investigated cell lines. The examples above, reveal that the tested MOF show differential toxicity and bioresponse in different effector cells tested, which indicate their differential suitability for specific medical purposes (11). In the study by Baati et al. (65), it has been demonstrated, that high doses $(220 \mathrm{mg} / \mathrm{kg}$ ) of Fe-MOFs (MIL-100, MIL-88A and MIL-88B-4CH 3 ) have shown no severe in viwo toxicity when administered intravenously to rats (65). 
Fig. 6 MOF cytotoxicity: (a) Dependence of fluorescence (in relative fluorescence units) on the concentration of Fe-MIL- $\mathrm{I} 0 \mathrm{I}-\mathrm{NH}_{2}$ contacted with $\mathrm{L} 929$ fibroblasts for 24 and $72 \mathrm{~h}$. M - milled and MU milled and ultrasonicated samples. C - control. (b) Photomicrographs of morphology of L929 fibroblasts after $24 \mathrm{~h}$ culture with addition of $0.625 \mathrm{mg} / \mathrm{ml}$ or $1.25 \mathrm{mg} / \mathrm{ml}$ of FeMIL-I OI-NH $\mathrm{NH}_{2}$ MOF. M - milled and $\mathrm{MU}$ - milled and ultrasonicated sample a

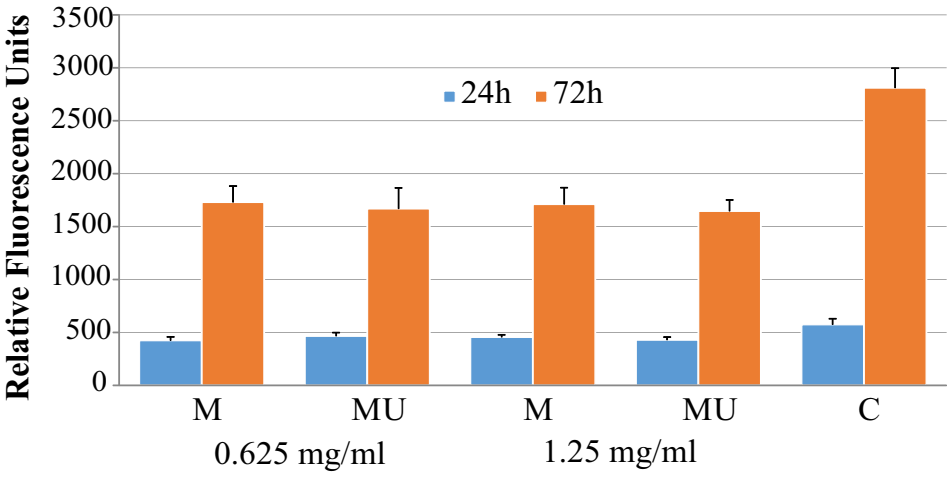

b

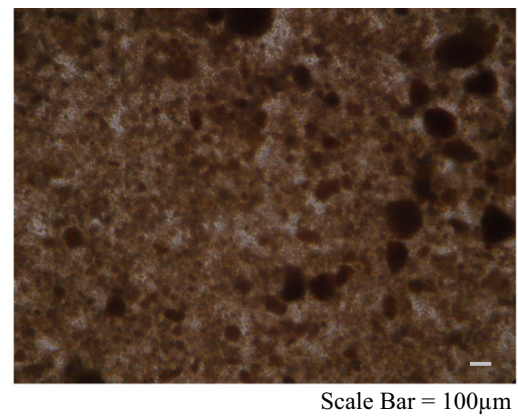

Fe-MIL-101-NH $-\mathrm{M} 1.25 \mathrm{mg} / \mathrm{ml}$

Fe-MIL-101- $\mathrm{NH}_{2}-\mathrm{M} 0.625 \mathrm{mg} / \mathrm{ml}$

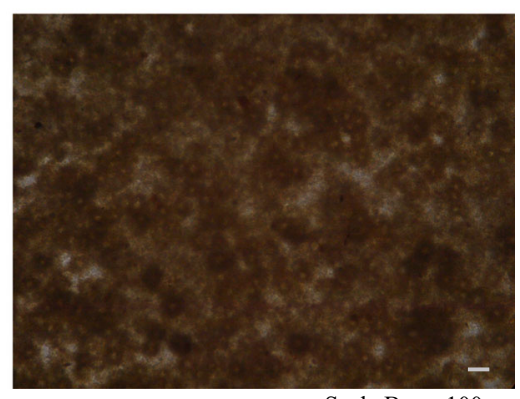

Scale Bar $=100 \mu \mathrm{m}$ Fe-MIL-101- $\mathrm{NH}_{2}-\mathrm{MU} 1.25 \mathrm{mg} / \mathrm{ml}$
Similarly to previous studies performed on various members of the MIL family, the results presented in the current study revealed the low cytotoxicity of investigated Fe-MIL101- $\mathrm{NH}_{2}$ material. It proves safety of Fe-MIL-101-NH $\mathrm{NH}_{2}$ as a potential drug carrier.

\section{CONCLUSIONS}

This work shows that Fe-MIL-101- $\mathrm{NH}_{2}$ Metal-Organic Framework can be an effective carrier for first-line anti-tuberculosis antibiotic - isoniazid. The developed material assured sustained drug release in opposite to fast dissolution of crystalline isoniazid powder. Additionally, magnetic resonance imaging and relaxometry on phantoms of the MOF system suspended in HPMC solution proved that proposed drug delivery system based on iron-MOF can also serve as the MRI contrast agent. These two features: drug delivery and imaging properties, combined in one carrier allow to classify Fe-MIL$101-\mathrm{NH}_{2}$ as theranostic agent.

According to performed in vitro cytotoxicity study the material was found to be safe. It has been observed that Fe-MIL101- $\mathrm{NH}_{2}$ particles were accumulated in the cell cytoplasmic area and were able to release drug inside cells, which makes them promising drug delivery system for local TB therapy. It can be expected that local drug action accomplished this way should increase therapy effectiveness, due to direct drug delivery to the MTB bacilli locations and diminish clinically assessed side effects of traditional systemic drug administration.

Presented results are the first step in development of inhalable drug delivery system based on iron-MOF. The obtained results suggest that it will be possible to optimize flow properties of the system to assure drug loaded MOF particles to reach alveoli level. Incorporation of other anti-TB drugs into MOF structure seems to be promising to ensure multitherapy and in consequence, prevent the development of 
MTB bacilli resistance. Illes et al. $(22,66)$ indicated the applicability of MOF in multi-drug therapy which has proven to be more effective than single-drug therapies in cancer treatment and in tuberculosis therapy is even obligatory.

In the advent of feasible translation of inhalable, pulmonary deposition monitoring to human (34), the application of MOF for extendedrelease inhalable system proposes the novel strategy for delivery of standard antimycobacterial agents combined with the monitoring of their distribution within the lung tissue.

\section{ACKNOWLEDGMENTS AND DISCLOSURES}

This work was supported by the National Science Center Poland [grant numbers 2014/15/B/ST5/04498 and 2016/21/N/NZ7/02663]; The IR measurements were carried out with the equipment purchased thanks to the financial support of the European Regional Development Fund in the framework of the Polish Innovation Economy Operational Program [contract number POIG.02.01.00-12-023/08].

Open Access This article is distributed under the terms of the Creative Commons Attribution 4.0 International License (http://creativecommons.org/licenses/by/4.0/), which permits unrestricted use, distribution, and reproduction in any medium, provided you give appropriate credit to the original author(s) and the source, provide a link to the Creative Commons license, and indicate if changes were made.

\section{REFERENCES}

1. Moghimi SM, Hunter AC, Murray JC. Nanomedicine: current status and future prospects. FASEB J. 2005;19(3):311-30.

2. Kim J, Piao Y, Hyeon T. Multifunctional nanostructured materials for multimodal imaging, and simultaneous imaging and therapy. Chem Soc Rev. 2009;38(2):372-90.

3. Parveen S, Misra R, Sahoo SK. Nanoparticles: a boon to drug delivery, therapeutics, diagnostics and imaging. Nanomedicine. 2012;8(2):147-66.

4. Formoso P, Muzzalupo R, Tavano L, De Filpo G, Nicoletta FP. Nanotechnology for the environment and medicine. Mini-Rev Med Chem. 2016;16(8):668-75.

5. Ahmed N, Fessi H, Elaissari A. Theranostic applications of nanoparticles in cancer. Drug Discov Today. 2012;17(17-18):928-34.

6. Sumer B, Gao J. Theranostic nanomedicine for cancer. Nano. 2008;3(2):137-40.

7. Funkhouser J. Reinventing pharma: the theranostic revolution. Curr Drug Discov. 2002;2:17-9.

8. Terreno E, Uggeri F, Aime S. Image guided therapy: the advent of theranostic agents. J Control Release. 2012;161(2):328-37.

9. Picard FJ, Bergeron MG. Rapid molecular theranostics in infectious diseases. Drug Discov Today. 2002;7(21):1092-101.

10. Freund R, Lachelt U, Gruber T, Ruhle B, Wuttke S. Multifunctional efficiency: extending the concept of atom economy to functional nanomaterials. ACS Nano. 2018;12(3):2094-105.
11. Wuttke S, Zimpel A, Bein T, Braig S, Stoiber K, Vollmar A, et al. Validating metal-organic framework nanoparticles for their Nanosafety in diverse biomedical applications. Adv Funct Mater. 2017;6(2). https://doi.org/10.1002/adhm.201600818.

12. Huxford RC, Della Rocca J, Lin W. Metal-organic frameworks as potential drug carriers. Curr Opin Chem Biol. 2010;14(2):262-8.

13. Wyszogrodzka G, Marszalek B, Gil B, Dorozynski P. Metal-organic frameworks: mechanisms of antibacterial action and potential applications. Drug Discov Today. 2016;21(6):1009-18.

14. Furukawa S, Reboul J, Diring S, Sumida K, Kitagawa S. Structuring of metal-organic frameworks at the mesoscopic/ macroscopic scale. Chem Soc Rev. 2014;43(16):5700-34.

15. Dong ZY, Sun YZS, Chu J, Zhang XZ, Deng HX. Multivariate metal-organic frameworks for dialing-in the binding and programming the release of drug molecules. J Am Chem Soc. 2017;139(40): 14209-16

16. Wuttke S, Lismont M, Escudero A, Rungtaweevoranit B, Parak WJ. Positioning metal-organic framework nanoparticles within the context of drug delivery - a comparison with mesoporous silica nanoparticles and dendrimers. Biomaterials. 2017;123:172-83.

17. Brown RW, Cheng YGN, Haacke EM, Thompson MR, Venkatesan R. Magnetic resonance imaging: physical principles and sequence design. Hoboken: John Wiley \& Sons; 2014.

18. Laurent S, Vander Elst L, Muller RN. Iron oxide nanoparticles as molecular MRI probes In: Merbach A, Helm L, Tóth E, editors. The chemistry of contrast agents in medical magnetic resonance imaging. Chinchester: John Wiley \& Sons; 2013. p. 442-443.

19. Aime S, Barge A, Gianolio E, Pagliarin R, Silengo L, Tei L. High Relaxivity contrast agents for MRI and molecular imaging. In: Bogdanov AA, Jr., Licha K, editors. Molecular Imaging. Ernst Schering Research Foundation Workshop. 49: Springer Berlin Heidelberg; 2005. p. 99-121.

20. Horcajada P, Chalati T, Serre C, Gillet B, Sebrie C, Baati T, et al. Porous metal-organic-framework nanoscale carriers as a potential platform for drug delivery and imaging. Nat Mater. 2010;9(2):1728.

21. Zimpel A, Preiss T, Roder R, Engelke H, Ingrisch M, Peller M, et al. Imparting functionality to MOF nanoparticles by external surface selective covalent attachment of polymers. Chem Mater. 2016;28(10):3318-26.

22. Illes B, Hirschle P, Baenert S, Cauda V, Wuttke S, Engelke H. Exosome-coated metal-organic framework nanoparticles: an efficient drug delivery platform. Chem Mater. 2017;29(19):8042-6.

23. Lismont M, Dreesen L, Wuttke S. Metal-organic framework nanoparticles in photodynamic therapy: current status and perspectives. Adv Funct Mater. 2017;27(14):1606314.

24. [Available from: http://www.who.int/tb/publications/global_ report/en/.

25. Gagandeep GT, Malik B, Rath G, Development GAK. Characterization of nano-fiber patch for the treatment of glaucoma. Eur J Pharm Sci. 2014;53(1):10-6.

26. Ormerod LP, Horsfield N. Frequency and type of reactions to antituberculosis drugs: observations in routine treatment. Tuber Lung Dis. 1996;77(1):37-42.

27. Gulbay BE, Gurkan OU, Yildiz OA, Onen ZP, Erkekol FO, Baccioglu A, et al. Side effects due to primary antituberculosis drugs during the initial phase of therapy in 1149 hospitalized patient's for tuberculosis. Respir Med. 2006;100(10):1834-42.

28. Bray PF. Isoniazid-induced acute toxic encephalopathy. Neurology. 1984;34(5):703.

29. Stettner M, Steinberger D, Hartmann CJ, Pabst T, Konta L, Hartung HP, et al. Isoniazid-induced polyneuropathy in a tuberculosis patient - implication for individual risk stratification with genotyping? Brain Behav. 2015;5(8):n/a. 
30. Kaur M, Garg T, Narang RK. A Review of emerging trends in the treatment of tuberculosis. Artif Cells Nanomed Biotechnol. 2014;44(2):478-84.

31. Kjellsson MC, Via LE, Goh A, Weiner D, Low KM, Kern S, et al. Pharmacokinetic evaluation of the penetration of Antituberculosis agents in rabbit pulmonary lesions. Antimicrob Agents Chemother. 2012;56(1):446-57.

32. Hickey AJ, Durham PG, Dharmadhikari A, Nardell EA. Inhaled drug treatment for tuberculosis: past progress and future prospects. J Control Release. 2016;240:127-34.

33. Pham DD, Fattal E, Tsapis N. Pulmonary drug delivery systems for tuberculosis treatment. Int J Pharm. 2015;478(2):517-29.

34. Wang HC, Sebrie C, Ruaud JP, Guillot G, Bouazizi-Verdier K, Willoquet $\mathrm{G}$, et al. Aerosol deposition in the lungs of spontaneously breathing rats using Gd-DOTA-based contrast agents and ultrashort Echo time MRI at 1.5 tesla. Magn Reson Med. 2016;75(2): 594-605.

35. Oakes JM, Scadeng M, Breen EC, Prisk GK, Darquenne C. Regional distribution of aerosol deposition in rat lungs using magnetic resonance imaging. Ann Biomed Eng. 2013;41(5):967-78.

36. Oakes JM, Breen EC, Scadeng M, Tchantchou GS, Darquenne C. MRI-based measurements of aerosol deposition in the lung of healthy and elastase-treated rats. J Appl Physiol. 2014;116(12): 1561-8.

37. Bauer S, Serre C, Devic T, Horcajada P, Marrot J, Ferey G, et al. High-throughput assisted rationalization of the formation of metal organic frameworks in the iron(III) aminoterephthalate solvothermal system. Inorg Chem. 2008;47(17):7568-76.

38. Hartmann M, Fischer M. Amino-functionalized basic catalysts with MIL-101 structure. Micropor Mesopor Mater. 2012;164:38-43.

39. Kim SY, Naskar D, Kundu SC, Bishop DP, Doble PA, Boddy AV, et al. Formulation of biologically-inspired silk-based drug carriers for pulmonary delivery targeted for lung Cancer. Sci Rep-UK. 2015;5:11878-91.

40. Terzano C, Allegra L, Alhaique F, Marianecci C, Carafa M. Nonphospholipid vesicles for pulmonary glucocorticold delivery. Eur J Pharm Biopharm. 2005;59(1):57-62.

41. Carvalho CDS, Daum N, Lehr CM. Carrier interactions with the biological barriers of the lung: advanced in vitro models and challenges for pulmonary drug delivery. Adv Drug Deliv Rev. 2014;75: 129-40.

42. Duncan GA, Jung J, Hanes J, Suk JS. The mucus barrier to inhaled gene therapy. Mol Ther. 2016;24(12):2043-53.

43. Rogers DF. Physiology of airway mucus secretion and pathophysiology of hypersecretion. Respir Care. 2007;52(9):1 134-49.

44. Pelfrene A, Cave MR, Wragg J, Douay F. In vitro investigations of human bioaccessibility from reference materials using simulated lung fluids. Int J Environ Res Public Health. 2017;14(2):112-27.

45. Son YJ, Horng M, Copley M, McConville JT. Optimization of an in vitro dissolution test method for inhalation formulations. Dissolut Technol. 2010;17(2):6-13.

46. Ferey G, Mellot-Draznieks C, Serre C, Millange F, Dutour J, Surble S, et al. A chromium terephthalate-based solid with unusually large pore volumes and surface area. Science. 2005;309(5743): 2040-2.

47. Horcajada P, Serre C, Vallet-Regi M, Sebban M, Taulelle F, Ferey G. Metal-organic frameworks as efficient materials for drug delivery. Angew Chem Int Ed. 2006;45(36):5974-8.

48. Taylor-Pashow KML, Della Rocca J, Xie Z, Tran S, Lin W. Postsynthetic modifications of Iron-carboxylate nanoscale metalorganic frameworks for imaging and drug delivery. J Am Chem Soc. 2009;131(40):14261-3.
49. Jin Z, Luan Y, Yang M, Tang J, Wang J, Gao H, et al. Imparting magnetic functionality to iron-based MIL-101 via facile Fe3O4 nanoparticle encapsulation: an efficient and recoverable catalyst for aerobic oxidation. RSC Adv. 2015;5(96):78962-70.

50. Ke F, Yuan Y-P, Qiu L-G, Shen Y-H, Xie A-J, Zhu J-F, et al. Facile fabrication of magnetic metal-organic framework nanocomposites for potential targeted drug delivery. J Mater Chem. 201 1;21(11): 3843-8.

51. Lebedev OI, Millange F, Serre C, Van Tendeloo G, Ferey G. First direct imaging of giant pores of the metal-organic framework MIL101. Chem Mater. 2005;17(26):6525-7.

52. Saifullah B, El Zowalaty ME, Arulselvan P, Fakurazi S, Webster TJ, Geilich BM, et al. Synthesis, characterization, and efficacy of antituberculosis isoniazid zinc aluminum-layered double hydroxide based nanocomposites. Int J Nanomedicine. 2016;1 1:3225-37.

53. Hirschle P, Preiss T, Auras F, Pick A, Volkner J, Valdeperez D, et al. Exploration of MOF nanoparticle sizes using various physical characterization methods - is what you measure what you get? CrystEngComm. 2016;18(23):4359-68.

54. Wu B, Lin X, Ge L, Wu L, Xu T. A novel route for preparing highly proton conductive membrane materials with metal-organic frameworks. Chem Commun. 2013;49(2):143-5.

55. Akyuz S, Akyuz T. FT-IR and FT-Raman spectroscopic studies of adsorption of isoniazid by montmorillonite and saponite. Vib Spectrosc. 2008;48(2):229-32.

56. Shaji J, Shaikh M. Current development in the evaluation methods of pulmonary drug delivery system. Indian. J Pharm Sci. 2016;78(3):294-306.

57. Azarmi S, Roa W, Lobenberg R. Current perspectives in dissolution testing of conventional and novel dosage forms. Int $\mathrm{J}$ Pharm. 2007;328(1):12-21.

58. Marques MRG, Loebenberg R, Almukainzi M. Simulated biological fluids with possible application in dissolution testing. Dissolut Technol. 201 1;18(3):15-28.

59. McConville JT, Patel N, Ditchburn N, Tobyn MJ, Staniforth JN, Woodcock P. Use of a novel modified TSI for the evaluation of controlled-release aerosol formulations. I. Drug Dev Ind Pharm. 2000;26(11):1191-8.

60. Adi H, Young PM, Chan HK, Salama R, Traini D. Controlled release antibiotics for dry powder lung delivery. Drug Dev Ind Pharm. 2010;36(1):119-26.

61. Haghi M, Traini D, Bebawy M, Young PM. Deposition, diffusion and transport mechanism of dry powder microparticulate salbutamol, at the respiratory epithelia. Mol Pharm. 2012;9(6): 1717-26.

62. May S, Jensen B, Wolkenhauer M, Schneider M, Lehr CM. Dissolution techniques for in vitro testing of dry powders for inhalation. Pharm Res. 2012;29(8):2157-66.

63. Gimenez-Marques M, Hidalgo T, Serre C, Horcajada P. Nanostructured metal-organic frameworks and their bio-related applications. Coord Chem Rev. 2016;307:342-60.

64. Grall R, Hidalgo T, Delic J, Garcia-Marquez A, Chevillard S, Horcajada P. In vitro biocompatibility of mesoporous metal (III; $\mathrm{Fe}, \mathrm{Al}, \mathrm{Cr}$ ) trimesate MOF nanocarriers. J Mater Chem B. 2015;3(42):8279-92.

65. Baati T, Njim L, Neffati F, Kerkeni A, Bouttemi M, Gref R, et al. In depth analysis of the in vivo toxicity of nanoparticles of porous iron(III) metal-organic frameworks. Chem Sci. 2013;4(4):1597607.

66. Illes B, Wuttke S, Engelke H. Liposome-coated Iron fumarate metal-organic framework nanoparticles for combination therapy. Nano. 2017:7(11):172-8. 